\title{
Performance of a bimanual load-lifting task by Parkinsonian patients
}

\author{
F VIALLET, ${ }^{*}$ J MASSION, $\dagger$ R MASSARINO, $\dagger$ R KHALIL* \\ From the Service de Neurologie, CHU La Timone, ${ }^{*}$ and Unité de Neurosciences Intégratives, $\dagger$ LNF, CNRS, \\ MARSEILLE France
}

SUMmaRY Normal subjects and Parkinsonian patients performed a bimanual load lifting task. In this task, one "postural" forearm, held in a horizontal position while supporting a $1 \mathrm{~kg}$ weight, was unloaded either by the experimenter's hand (imposed unloading) or by the subject's other hand in response to a tone burst (voluntary unloading). The variables recorded were reaction time (RT: time interval between the tone and beginning of unloading) and movement time (MT: duration of the change in force measured by a force platform on the "postural" forearm). Elbow angle changes were also measured with a potentiometer. The EMG activity from brachioradialis of the "postural" arm and that from the biceps of the "active" arm were recorded. The Parkinsonian patients showed an increase in both RT and MT and an impairment of the co-ordination between movement and posture which was reflected in an increase in amplitude of the elbow rotation after voluntary unloading. Moreover, the decrease in EMG activity in the brachioradialis of the postural arm during unloading was less in Parkinsonian patients than in the normal group. This disorder of postural command was often accompanied by a lack of anticipatory EMG changes. Comparison between treated and non-treated patients showed that dopamine agonists brought about recovery of both RT and MT but did not improve postural co-ordination. The co-ordination was less impaired when the voluntary unloading was performed by the preferred hand. Several hypotheses are discussed concerning the mechanism underlying this impaired co-ordination.

Performance of a voluntary movement by one body segment is usually accompanied by an adjustment of posture aimed at preventing the imbalance which would otherwise occur. ${ }^{1}$ Posture and movement have been shown to be co-ordinated in several situations. Under near-static conditions, for instance, Babinski ${ }^{2}$ observed that backward bending of the body is accompanied by flexion of the knees, restricting displacement of the vertical projection of the centre of gravity to within the area delimited by the feet on the ground. Similarly, raising of the arms forwards leads to a compensatory backward displacement of the trunk. ${ }^{3}$ Under dynamic conditions, when the voluntary movement is a fast one, the postural adjustment command usually precedes the actual movement. An

Address for reprint requests: Dr J Massion, LNF-U3-CNRS 31, Chemin Joseph Aiguier 13402 MARSEILLE CEDEX 9 FRANCE

Received 22 July 1986 and in revised form 4 February 1987. Accepted 11 February 1987 anticipatory postural adjustment has been shown to occur in the lower limbs when a voluntary movement is performed by the upper limbs. ${ }^{45}$ This postural adjustment compensates in advance for the effects the voluntary movement will have on the body's overall equilibrium. ${ }^{6}$ Its adaptation to changes in the postural constraints or in the movement parameters has been described. ${ }^{78}$ In a situation involving a local disturbance of an upper limb, early postural adjustments have been observed to occur in the limb segments responsible for postural maintenance. ${ }^{9}$ The postural command is said in such cases to be anticipatory because the electromyographic activity corresponding to the postural adjustment occurs prior to the actual mechanical changes in posture resulting from the initial disturbance. ${ }^{10}$

There exists a comparable approach to the problem of posture and movement co-ordination, based on the use of a bimanual unloading task. Hugon et al ${ }^{11}$ have shown that the position of a weight-supporting forearm changes very little when the weight is taken over by the contralateral hand. EMG recordings have 
shown that an inhibition in the brachioradialis muscle precedes the onset of the change in force caused by the voluntary unloading by the opposite hand, indicating an anticipatory postural adjustment. ${ }^{12}$

It has been reported that in Parkinson's disease, these anti-gravitational postural adjustments are often impaired. ${ }^{3}$ Traub et al ${ }^{13}$ have shown that in Parkinsonian patients, an externally applied disturbance of the upper limb is often accompanied by a defective anticipatory postural adjustment at sural triceps level. Parkinson's disease has also been reported to involve a disturbance of the variables characteristic of voluntary movements, such as the lengthening of reaction time and movement time. ${ }^{14-16}$

The aim of the present study was to analyse postural and kinetic co-ordination in a bimanual unloading task. We attempted to assess the impairment of postural adjustment displayed by one arm in association with a voluntary movement performed by the other. Furthermore, two additional problems were investigated in connection with the Parkinsonian patients. The first specifically concerned the effects of treatment with dopamine agonists; and the second concerned the effects of handedness in the bimanual task studied.

\section{Material and methods}

In this study, the term "postural arm" is used to denote the arm which is loaded and unloaded, and the term "active arm" to denote the arm performing the voluntary unloading movement.

\section{Experimental arrangement}

The experimental arrangement was the same as that described in previous papers. ${ }^{11}{ }^{12}$ The subjects were seated, with their gaze fixed horizontally on a distant point in front of them. Their postural arm was maintained vertically beside their body, by a semi-circular holder placed above the elbow (fig 1). They were requested to maintain the forearm in a horizontal position, holding the wrist semi-prone. With this forearm, subjects had to hold up a little tray $(\mathrm{F})$ fitted with strain gauges on which was placed a $1 \mathrm{~kg}$ weight $(\mathrm{L})$. A linear potentiometer $(\mathrm{P})$ was placed on the rotation axis of the elbow joint.

In the imposed unloading situation (A), the experimenter unpredictably removed the weight.

In the voluntary unloading situation (B), the subjects themselves were instructed to take the weight with the other hand when they heard an auditory signal (BIP).

Ten to 15 tests were run in each of the two situations. The subjects' two arms were alternated symmetrically as to which arm performed the voluntary movement and which supported the weight.

\section{Measurements}

The parameters recorded are given in fig 2 . The change in force was measured by the strain gauges fitted to the tray.
The angular displacement of the forearm was measured by the potentiometer. The EMG recordings were obtained by means of pairs of surface electrodes placed 2 to $3 \mathrm{~cm}$ apart on the main part of the brachioradialis muscle of the postural arm and that of the biceps muscle of the active arm. The amplified EMG activity was rectified and integrated with a time constant of $10 \mathrm{~ms}$. All the data (change in force, angular displacement of the forearm, EMG activities) were stored and analysed on a computer with a sampling frequency of $200 \mathrm{~Hz}$. The signals were averaged taking the onset of the change in force as the starting (reference) point from which the traces were aligned.

\section{Experimental variables}

The variables of interest in the voluntary movement were as follows: the reaction time ( $\mathrm{RT})$, which was defined as the time elapsing between the onset of the auditory signal and the beginning of the change in force, and the movement time (MT), which corresponded to the duration of unloading or the time elapsing between the beginning and the end of the change in force from 1 to $0 \mathrm{~kg}$.

The co-ordination between posture and movement was measured indirectly, taking as a quantitative index the relative amplitude of the elbow rotation (Ar), which was calculated in terms of the following relation between the angular displacements obtained after voluntary (Av) and imposed (Ai) unloading:

$$
\operatorname{Ar}(\%)=\mathrm{Av} / \mathrm{Ai} \times 100
$$

The EMG activity of the brachioradialis muscle in the postural arm was measured using a scale on which zero activity (level 0 ) was taken to be that obtained with the forearm resting on a support, after waiting till the EMG became silent, and maximum activity (level $1 \cdot 0$ ) that obtained with the horizontal forearm supporting a $1 \mathrm{~kg}$ load. The EMG inhibition observed when unloading occurred was then plot-
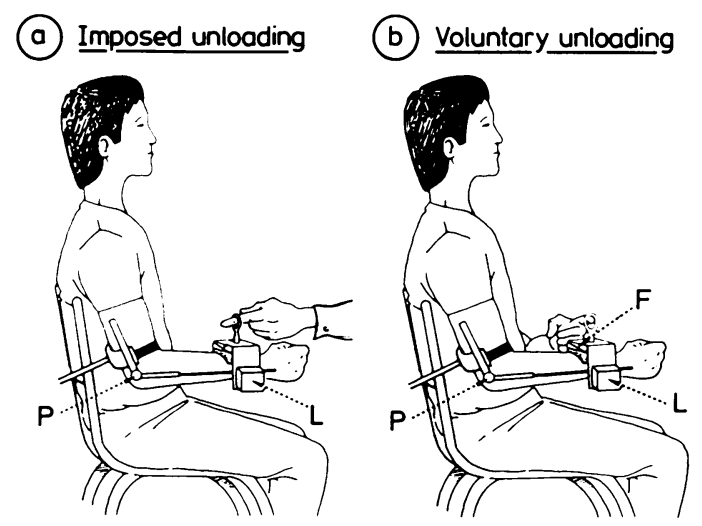

Fig 1 Experimental arrangement. The set-up includes a strain-gauge tray $(F)$ placed on the subject's forearm with the load $(L)$. The forearm position was measured by means of a linear potentiometer $(P)$. The $1 \mathrm{~kg}$ load placed on the strain-gauge tray could be relieved in two different ways, as follows: $A=$ removed by the investigator (imposed unloading); $B=$ removed by the subject's other hand (voluntary unloading). 


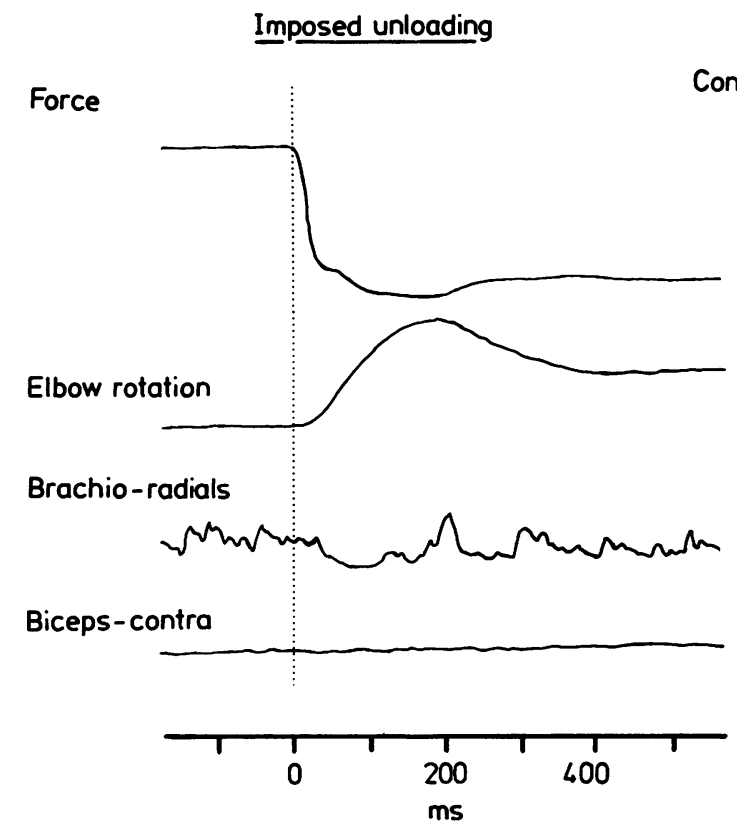

\section{Voluntary unloading}

Control

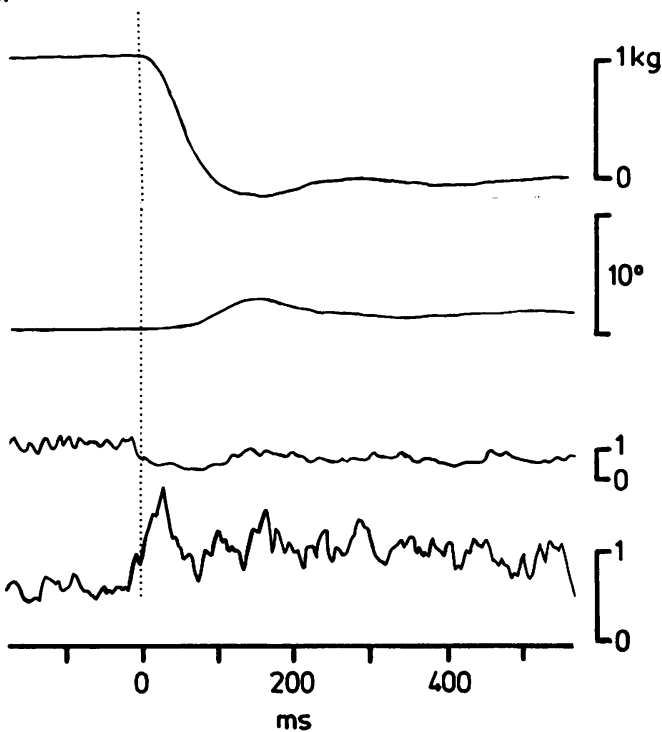

Fig 2 Recordings on a normal subject under conditions $A$ (imposed unloading) and B (voluntary unloading). The parameters recorded are as follows, from top to bottom: force, angular displacement of the forearm, integrated EMG activities of the brachioradialis muscle of the postural arm and the contralateral biceps. Each trace corresponds to the mean for 10 tests. Calibration of the EMG: zero = level of activity when the forearm was resting on a support and the muscle silent; $1 \cdot 0=$ level of activity with the forearm maintained in horizontal position supporting a $1 \mathrm{~kg}$ weight.

ted as a value on this scale. This value was the lowest level which was measured over a time interval ranging from $100 \mathrm{~ms}$ before onset of the change in force to $150 \mathrm{~ms}$ after onset of the change in force.

\section{Choice of subjects}

A group of 14 Parkinsonian patients was studied. All the subjects were right-handed. The subjects' individual characteristics (age, sex, duration of the disease, disability assessment according to Hoehn and Yahr's scale, ${ }^{17}$ method of treatment) are given in the table.

Their average age was 64.4 years (range 54 to 73). The mean duration of the disease was 8.2 years (range $1-28$ years). The mean disability score was 2.5 (one patient in stage I, five in stage II, eight in stage III).

Nine patients were being treated with dopamine agonists (levodopa associated with a DOPAdecarboxylase inhibitor, or bromocriptine or piribedil); the other five were undergoing no treatment at the time of the study.

The RTs were not measured in two patients. Also, EMG analysis could not be interpreted in three patients with particularly pronounced tremor. A control group of five normal subjects was set up from among hospital patient volunteers with no cerebral lesions. These patients were also righthanded. Their average age was 60.4 years (range 45-77 years).

\section{Results}

\section{Qualitative study}

Figure 2 illustrates the results obtained with a normal subject. In the imposed unloading situation, the change in force was followed by an upward angular displacement of the elbow. In the brachioradialis muscle of the postural arm, an inhibition of EMG activity was observed with a latency of $30 \mathrm{~ms}$ in relation to the onset of the change in force. In the voluntary unloading situation, the amplitude of the angular displacement of the elbow was found to be smaller than under imposed unloading. The inhibition of the EMG activity of the postural brachioradialis muscle was practically synchronous with the increase in EMG activity observed in the "active" arm biceps. This anticipatory inhibition was found to occur about $20 \mathrm{~ms}$ before the change in force.

Figure 3 gives the data obtained with a Parkinsonian patient. In the imposed unloading situation, the results (angular displacement of the elbow, reflex inhibition of EMG activity in the brachioradialis muscle) were comparable to those produced by 
normal subjects. For example, the mean value of the elbow rotation (Ai) in the control subjects was $6^{\circ}$ versus $6^{\circ} 5^{\prime}$ in the Parkinsonian patients. The stiffness of some patients did not lead to any marked change in $\mathrm{Ai}$. Under voluntary unloading, the duration of the change in force was longer than in the normal group, for the Parkinsonian patient represented in fig 3. This was associated with a larger displacement of the elbow than that observed in normal subjects in the same situation; moreover, inhibition of the EMG activity in the postural brachioradialis muscle was found to occur only after the onset of the change in force, presumably reflecting the absence of any anticipatory mechanisms.

\section{QUANTITATIVE STUDY}

\section{Overall data}

The results are given in figs 4 and 5 . The two arms were tested alternately as to which arm supported the load and which performed the voluntary movement, so that two sets of results were obtained for each subject. Statistical comparisons were carried out on the various experimental variables. Mean values and standard deviations were calculated. Because of the
non-Gaussian distribution of some series, non parametric methods (Mann-Whitney's U-test) were also used in statistical comparisons.

The mean values of the experimental variables (RT, MT, Ar) are presented for both normal subjects and Parkinsonian patients in the form of histograms (fig 4). The mean RT values were higher in the Parkinsonian group $(232.4 \pm 55.8 \mathrm{~ms})$ than in the normal subjects $(183.6 \pm 23.8 \mathrm{~ms})$. This was also the case with the mean MT values, which were higher in the Parkinsonian patients $(274.5 \pm 73.1 \mathrm{~ms})$ than in the normal subjects $(211.9 \pm 56.6 \mathrm{~ms})$. The relative amplitude of elbow rotation (Ar) was also conspicuously larger in the Parkinsonians $(51.9 \pm 20.5 \%)$ than in the normal group (30.8 $\pm 7 \cdot 3 \%)$. These results thus confirm that RT, MT and Ar were significantly higher in the Parkinsonian than in the normal subjects.

Figure 5 gives the individual lowest values of the EMG activity level during the inhibitory phase (dots) in the brachioradialis muscle in situations A (imposed unloading) and B (voluntary unloading) for each of the normal subjects and each Parkinsonian patient. In the voluntary unloading situation, the mean value of the level of EMG activity during the inhibitory
Imposed unloading

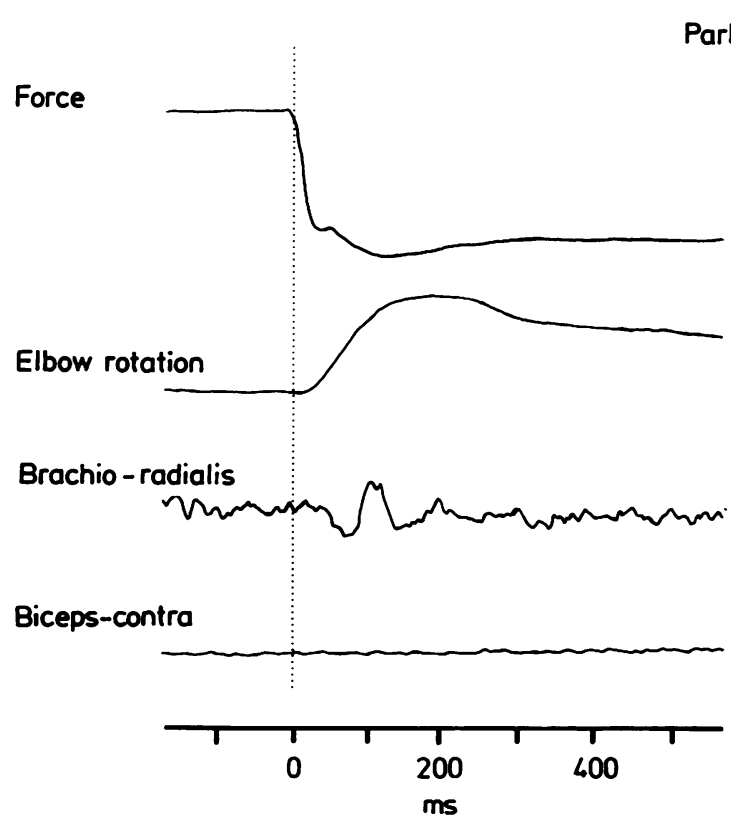

Voluntary unloading

Parkinson

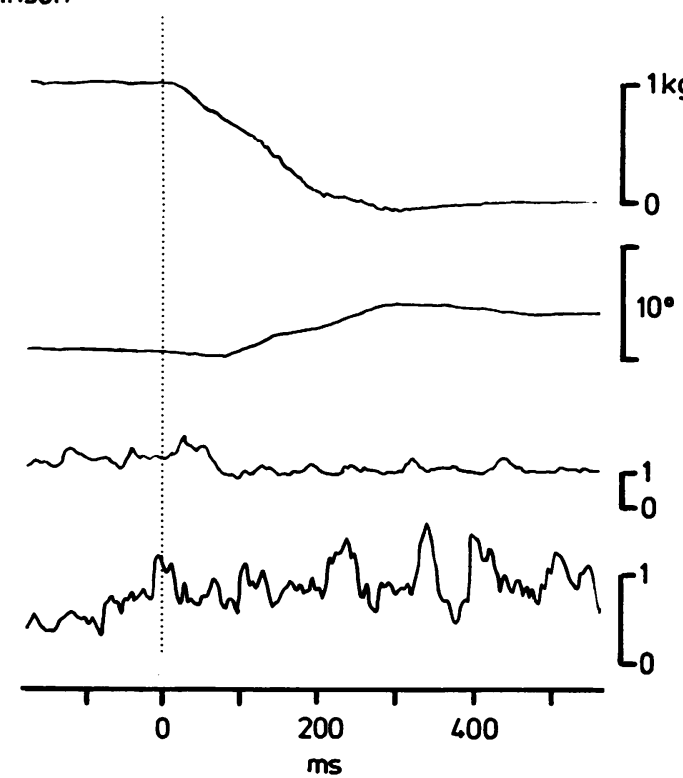

Fig 3 Recordings on a Parkinsonian patient under conditions $A$ (imposed unloading) and B (voluntary unloading). The following parameters were recorded, from top to bottom: force, angular displacement of the forearm, integrated EMG activities of the brachioradialis muscle of the postural arm and the contralateral biceps. Traces correspond to the mean for 10 tests. EMG calibration as in fig 2. 


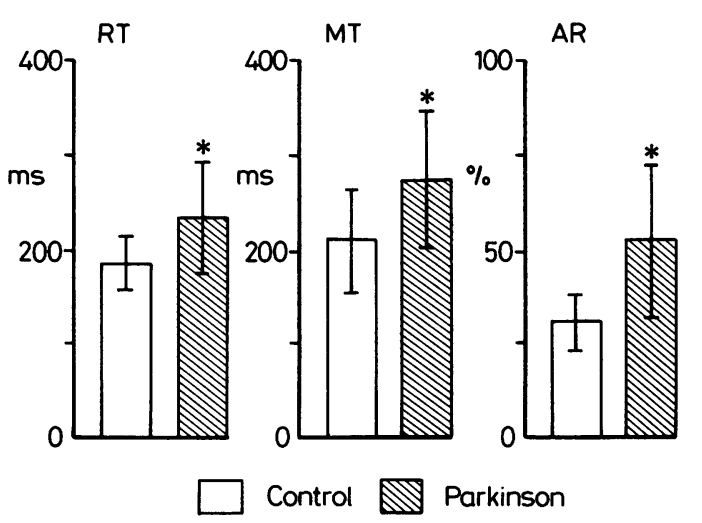

Fig 4 Experimental variables. $R T=$ reaction time $(\mathrm{ms})$, $M T=$ movement time $(\mathrm{ms}), A r=$ relative amplitude of elbow rotation $(\%) .{ }^{*}=p<0 \cdot 05$. The histograms show mean values and standard deviations obtained by the group of normal subjects (white) and by the Parkinsonian patients (shaded).

phase (thick bar) was higher in the Parkinsonians $(0.52 \pm 0.30)$ than in the normal subjects $(0 \cdot 13 \pm 0 \cdot 08)$. This was also the case under imposed unloading, where these mean values were $0 \cdot 26 \pm 0 \cdot 20$ in the Parkinsonians and $0 \cdot 10 \pm 0 \cdot 11$ in the normal subjects. Inhibition of the EMG activity in the brachioradialis muscle was thus significantly less in the Parkinsonians than in the normal group under both voluntary and imposed unloading. Moreover this difference was associated under voluntary unloading with a loss of the anticipatory character of the EMG response (empty circles) in more than half of the traces analysed (13 out of 22$)$.

Further statistical analysis was carried out on the Parkinsonian group in order to establish what, if any, effects were due to administration of anti-Parkinson drugs and the patients' handedness.

\section{Effects of anti-Parkinson drugs}

Here again statistical analysis was carried out with non-parametric methods (Mann-Whitney's U-test) to compare the group of patients receiving no treatment $(\mathrm{N}=5)$ with those under treatment $(\mathrm{N}=9)$. The results obtained (fig 6) show that the mean RT values were significantly lower in the treated patients $(209.6 \mathrm{~ms})$ than in those receiving no treatment $(264.3 \mathrm{~ms})$. This was also found to be the case with the MTs which were lower in the treated $(247.3 \mathrm{~ms})$ than the non-treated patients $(323.4 \mathrm{~ms})$. No significant difference in the mean Ar values was observed, however, between the treated $(55.9 \%)$ and non-treated groups $(44 \cdot 7 \%)$.
Effects of handedness

The results are given in fig 7. Statistical comparison between the two sets of values obtained when the voluntary movement was performed with the right vs. left hand showed no significant differences in the RT, MT or Ar in either normal subjects or non-treated Parkinsonians. In the patients undergoing treatment, however, no significant change in RT was observed, but the MT and Ar values were significantly higher when the voluntary movement was performed with the left hand $(273.7 \mathrm{~ms}$ and $64.6 \%$, respectively) than with the right hand $(220.8 \mathrm{~ms}$ and $47.2 \%$, respectively). The anti-Parkinson treatment thus gave rise to a difference in performance level between the two hands. The performance of the voluntary movement improved more markedly in the case of the preferred hand; conversely, coordination deteriorated even more when the lifting was performed by the nonpreferred hand.

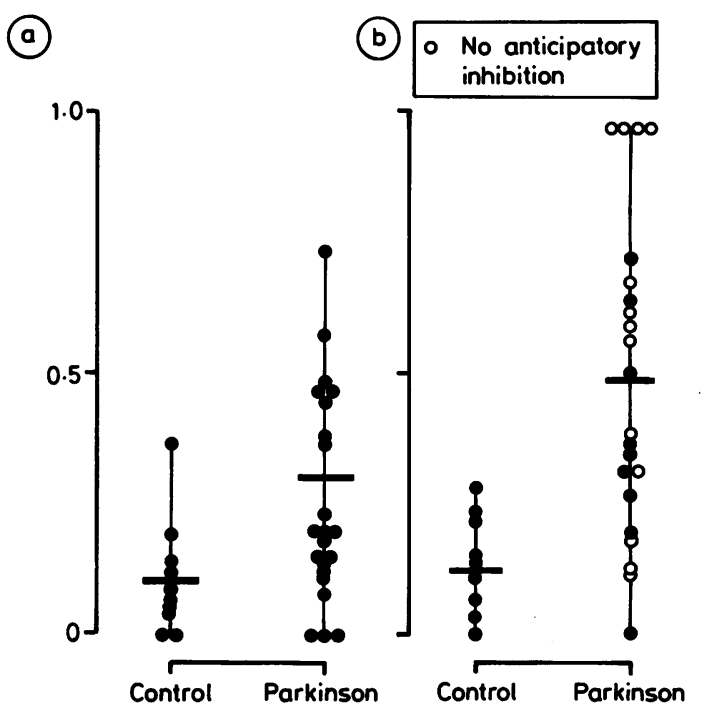

Fig 5 EMG inhibition in the brachioradialis muscle of the postural arm during imposed unloading $(A)$ and voluntary unloading $(B)$. Each dot represents, for one of the subjects arms, the mean value over 10 to 15 tests of the lowest level of EMG activity during the unloading phase, corresponding to the level of inhibition. Horizontal bars represent the mean of all dots for the normal group and the Parkinsonian group. (Empty circles are used instead of dots to indicate the loss of the anticipatory character of EMG inhibition). Scale of EMG activity-level 0: activity recorded with forearm at rest,

-level 1.0: activity recorded with horizontal forearm supporting a $1 \mathrm{~kg}$ load. 


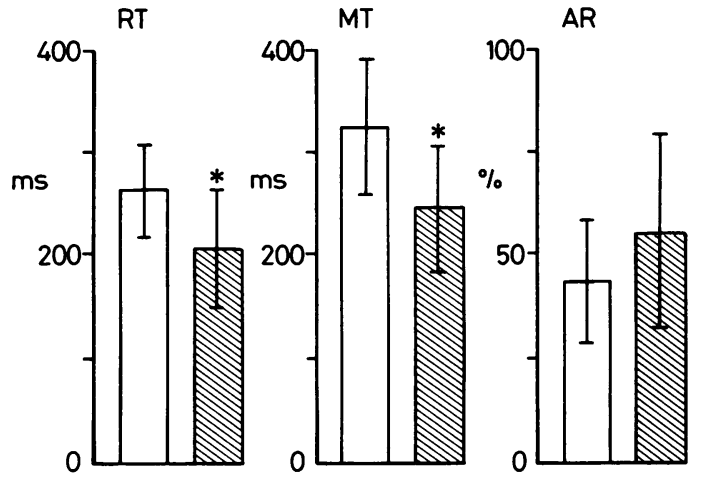

Treated $\square$ Not treated

Fig 6 Comparison between treated and non-treated Parkinsonians. Experimental variables as in fig 4. The histograms show mean values and standard deviations obtained by the group of patients undergoing no treatment (white) and those under treatment (shaded).

\section{Discussion}

Among the various techniques for studying postural adjustments in Parkinsonians that have been described in the literature, the analysis of co-ordination between posture and movement presented here has a special feature in that it deals with a voluntary rather than a passive movement.

It is proposed to discuss the results obtained in this study on a group of Parkinsonian patients, first from the point of view of the variables of the voluntary movement, and secondly from that of the postural command itself.

\section{Variables of the voluntary movement}

The RT and MT data show clearly that the initiation and performance of the voluntary movement, namely the lifting of a $1 \mathrm{~kg}$ load with one hand, take longer to accomplish in Parkinsonian patients than in normal subjects. This is consistent with classical studies on voluntary movements in subjects suffering from Parkinson's disease (cf ref 18). The motor tasks used in previous studies have been either the tracking of a visual target ${ }^{1419-22}$ or single joint movements of the upper limbs triggered by a visual or proprioceptive signal. ${ }^{1523}$ If one takes the reaction times recorded here in the voluntary unloading movement triggered by an auditory signal, the difference between Parkinsonian patients and normal subjects can be seen to be fairly slight, quantitatively speaking. The interindividual variability was higher, however, among the Parkinsonians than among the normal subjects. This may be due, as we shall suggest below, to the effects of
anti-Parkinson drugs on patients undergoing treatment. The slowing down of the voluntary movement which was observed in the Parkinsonian patients might be due to the amplitude of the biceps and brachioradialis muscle EMG activity being reduced in the arm performing the movement. The recording given in fig 3 illustrates the lengthening of unloading time observed in these patients, as well as the decrease in biceps EMG activity in the active arm. Previous EMG analyses of single-joint movements in Parkinsonian patients have shown that a reduction in the "energising" and a disturbance of the timing of the muscular contraction occur. ${ }^{23}$ This disorder is presumably caused by insufficient coding by the central

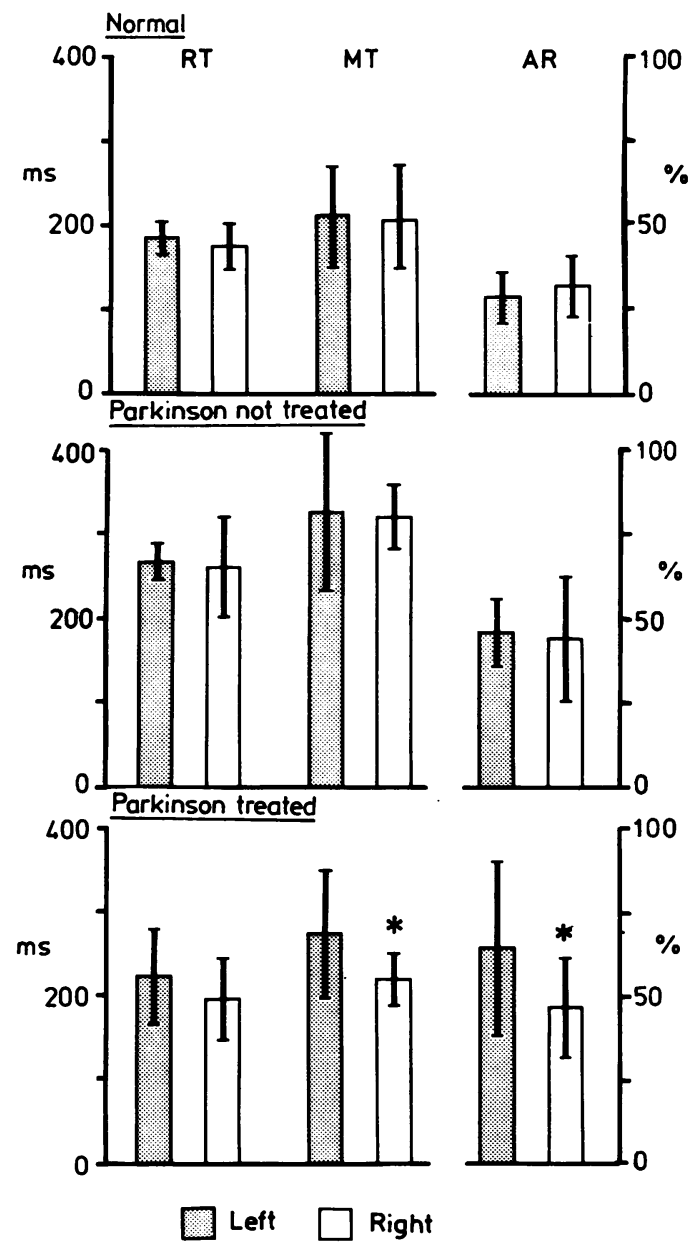

Fig 7 Comparison between left and right voluntary hands. Experimental variables as in fig 4. The histograms show mean values and standard deviations obtained with the left (shaded) and right (white) voluntary hands. 
Table Characteristics of the Parkinsonian patients tested

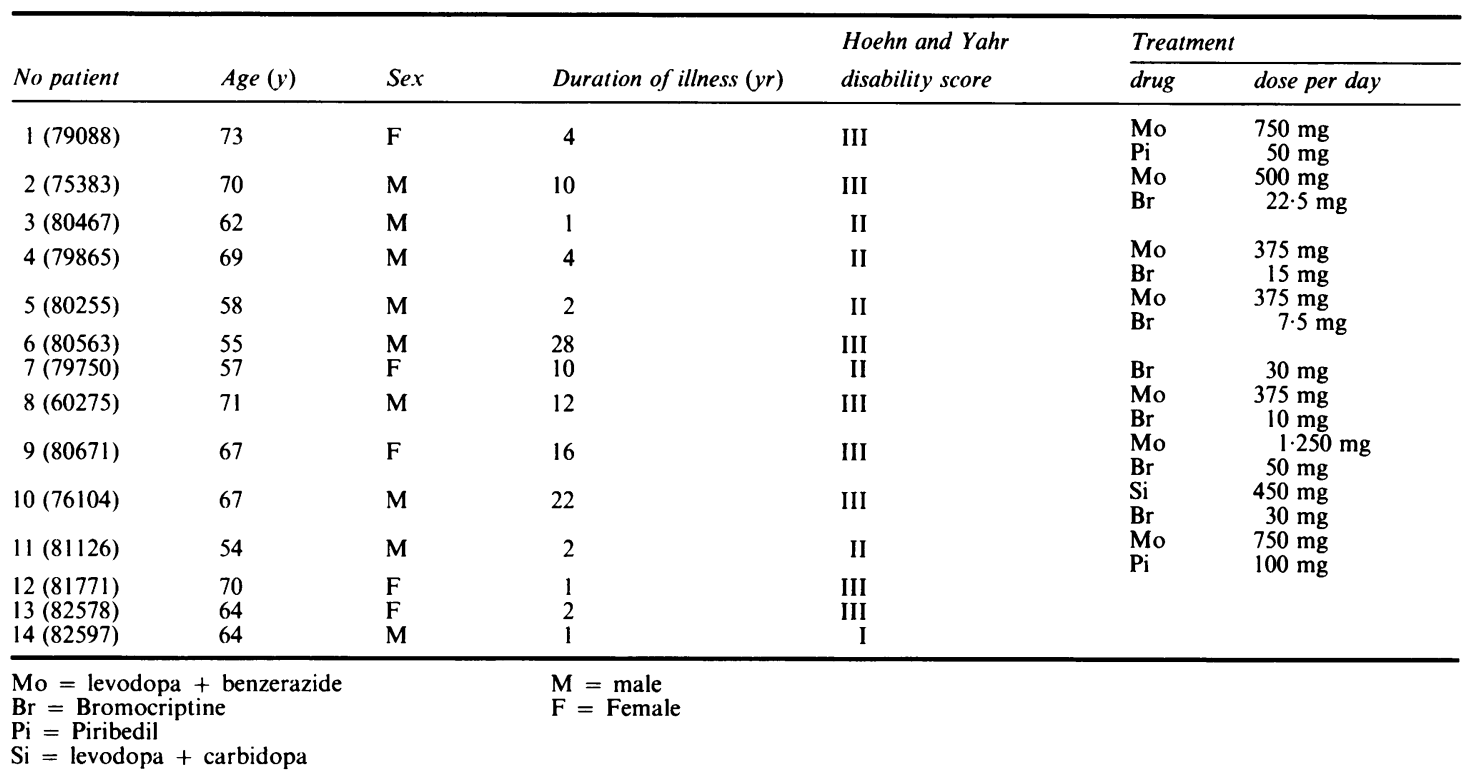

command of the force necessary to raise the weight. Parkinsonian subjects certainly have difficulty in performing fast movements, especially when visual control is lacking, because of the "motor plan" disorders involved, ${ }^{24}$ so that it is quite likely that they may choose a slower strategy to perform the requisite movement. This suggests that Parkinsonians may tend to control their movements through a feed-back rather than a feed-forward mode, which will have repercussions on the organisation of the associated postural adjustments.

\section{Postural command}

According to a hypothesis put forward by Hugon et al, ${ }^{11}$ the triggering of a voluntary movement in one arm can release a near-simultaneous postural adjustment of the other arm. This co-ordination between movement and posture limits the angular displacement of the forearm which follows unloading, since an anticipatory inhibition of flexor muscle activity occurs in the postural arm. The postural command can be analysed through the anticipatory character and the level of the EMG inhibition.

In the present study, the co-ordination between posture and movement was gauged indirectly from an index, namely the relative amplitude of the elbow rotation $\operatorname{Ar}$ (see Methods). The results obtained show that the Ar values were significantly higher in the group of Parkinsonian patients than in the normal subjects. This difference in Ar results from an increase in the angular displacement of the forearm under vol- untary unloading, in relation to the angular displacement of the forearm under imposed unloading. It indicates that co-ordination between movement and $\bar{\alpha}$ posture is disturbed in Parkinsonian patients. By analysing the EMG activity in the brachioradialis of the postural arm, it was possible to discern moreo clearly how the postural command was impaired: the anticipatory EMG inhibition consistently observed prior to the change in force in the recordings from the normal subjects ( 10 out of 10 ) was lacking in more than half of the recordings obtained with the Parkinsonian group (13 out of 22). This indicates a trend in Parkinsonians to shift from a feedforward to a feedback mode of control. Furthermore, the inhibition of EMG activity was significantly less in the Parkinsonian patients than in the normal subjects. This probably means that these patients are less able to compensate in advance for the forearm rotation. However, in rigid patients with higher levels of initial contraction the possibility that real extent of inhibition may have been underestimated here cannot be excluded.

The findings that co-ordination between posture and movement is impaired in Parkinsonian patients confirms previous clinical data. ${ }^{3}$ Traub et al ${ }^{13}$ have studied the postural adjustment at sural triceps level resulting from an external disturbance applied to an upper limb and reported a loss of postural anticipation in 12 Parkinsonians out of the 29 studied. Moreover, the mean value of the change in the EMG activity of the sural triceps was significantly less in the 
Parkinsonian than in the normal subjects. It should be pointed out, however, that the reflex postural adjustment studied by Traub et $a l^{13}$ is not strictly comparable with that associated with the voluntary movement analysed in the present study.

How to interpret this impaired co-ordination between posture and movement in Parkinsonian patients is still largely a matter of conjecture as far as our present understanding of the bimanual unloading task is concerned. Two hypotheses can be suggested, however, to explain how this co-ordination is organised. According to the first, the systems serving to command the voluntary movement and the postural adjustment can be thought of as being separate; they might however be synchronised at some supraspinal level which has still to be determined. According to the second hypothesis, a single command may operate at spinal level, co-ordinating the voluntary movement with the postural stance. Triggering of the voluntary movement might generate an internal message of the collateral type involving a coding of the specific parameters of the voluntary movement, so that an appropriate command might be delivered to the postural arm. Some experimental data on bimanual unloading in normal subjects argue in favour of this idea. For example, the anticipatory character of the postural command was no longer observed when subjects had to trigger the unloading voluntarily by switching off an electromagnet. ${ }^{12}$ If, however, subjects voluntarily triggered the unloading by means of a force command that alleviated the load indirectly, postural anticipation re-appeared. ${ }^{25}$ Both normal and Parkinsonian subjects were found in our study to develop the same force $(1 \mathrm{~kg})$ during the voluntary movement. If one takes the duration of unloading (MT) into account, however, the Parkinsonians were found to take longer so that the slope of their unloading curve (df/dt) was not as steep as that of the normal subjects. The decrease in this slope to below a hypothetical threshold (which still remains to be specified, and may depend on certain experimental conditions) might be accompanied by the loss of the anticipatory character of the postural command. In order to check on this interpretation, the MT values were compared between two groups of Parkinsonians, in one of which the brachioradialis EMG recordings showed signs of postural anticipation, and in the other, none. The mean MT value was significantly higher $(p<0.05)$ in the "no anticipation" group $(334.6 \pm 93.0)$ than in the "anticipation" group (262.2 $\pm 97 \cdot 1 \mathrm{~ms})$. A complementary, parallel interpretation might be proposed, based on the strategy or "motor plan" concept. ${ }^{24}$ As Friedli has observed, ${ }^{8}$ the anticipatory character of such postural adjustments suggests that they may be programmed in advance. The loss of the anticipatory character of postural command in Parkinsonian patients might thus be due to the fact that they use a different strategy, involving a feed-back mode of postural control to compensate a posteriori for the disturbance caused by the voluntary movement, instead of the feed-forward control which normally accompanies voluntary movements.

\section{Effects of anti-Parkinson drugs}

The data obtained in the present study show that in the group of patients undergoing no treatment, the duration of the voluntary movement was longer than in patients taking anti-Parkinson drugs. Furthermore it can be seen that the RTs and the MTs of patients undergoing treatment were practically the same as those of the normal subjects. These findings serve first of all to confirm the efficiency of dopaminergic agonists as a means of treating the akinesia symptomatic of Parkinson's disease. They help to improve in particular the reaction time and the time required to perform voluntary movements. ${ }^{142627}$ This improvement was quantitatively more conspicuous in the case of the MTs than in that of the RTs. ${ }^{28}$ The relative amplitude of elbow rotation (Ar) did not, however, show a similar return to normal levels. Paradoxically, the mean Ar value was even slightly higher with the treated patients, although the difference between treated and untreated patients was not actually significant. It might be suggested that although a sufficiently high level was reached by the force command in most of the patients receiving treatment, regulation of the associated postural command still remained impaired. It is also possible that Parkinsonians, despite efficient treatment, may have lost the ability to control postural adjustments by means of a feed-forward mode and that they continue to use a feedback postural command strategy although their performance of the voluntary movement has apparently improved. This interpretation suggests that there may exist a kind of hierarchy of motor disorders in Parkinson's disease, which depends on the complexity of the movement to be performed. From this point of view, which is analogous in several respects to the "motor plan" concept ${ }^{24}$ the voluntary movement consisting of raising a load with one hand while seated is apparently a fairly simple one. The loadlifting would be more complex if the subject were required to remove the load with one hand while keeping the other unloaded arm horizontal which would involve an additional postural constraint. The level of complexity can in fact be reduced for normal subjects by training, so that the postural command associated with the voluntary movement becomes to some extent automatic. The postural command can then be organised during preparation of the movement through a feed-forward mode which parallels 
the initiation of the voluntary movement. ${ }^{8}$ In Parkinsonian patients, the loss of "associated movements" seems to constitute an early symptom of the disease in the course of which gradual slowing down of voluntary movement occurs. The postural adjustments we have assimilated here to associated movements seem to present a high level of complexity because of the difficulty Parkinsonians have in performing automatic movements, that is, movements organised according to a feed-forward mode. ${ }^{29}$ The recovery of motor abilities obtained by administration of dopamine agonist drugs might apply only to voluntary movements, depending on their degree of complexity, and might not have sufficiently far-reaching effects to also improve the associated postural adjustments.

\section{Effects of handedness}

The bimanual task used in this study corresponds to a fairly common motor situation in everyday life, obviously involving a slight lateralisation factor which depends on the subject's handedness. The coordination between movement and posture involved in this situation may be listed, so to speak, in a "repertoire" of stereotyped movements. In terms of complexity, this task probably comes quite low on the list with normal subjects, who produced identical results whichever hand performed the voluntary movement. This was not the case with the Parkinsonian patients, whose results were less impaired when the task was performed with the preferred hand. More specifically, the results of patients undergoing no treatment showed symmetrical impairment on both sides; whereas in patients under treatment, the movement time and the postural command showed less impairment when the voluntary movement was performed with the preferred hand than with the contralateral hand. The level of complexity can thus be said to have been lower when the task was performed with the preferred hand. In fact, it may be that the impaired processes underlying automatic bimanual co-ordination are more easily recovered when the voluntary movement is carried out by the preferred hand.

\section{Conclusion}

Analysis of the co-ordination between posture and movement in Parkinsonian subjects performing a bimanual task is an original, fairly straightforward approach. The results obtained confirm that the initiation and execution of the voluntary movement studied here slow down during Parkinson's disease. The postural command associated with the voluntary movement was furthermore found to be impaired both qualitatively (loss of its anticipatory character) and quantitatively (decrease in EMG inhibition in the flexor muscles of the postural arm), which caused an inability to maintain the position of the unloaded forearm, as shown by the anomalous increase in the angular displacement. By conducting further investigations on a larger number of patients, it should be possible in the future to show that this can be a useful method for quantitatively assessing motor disorders in Parkinsonians, so as to be able to observe the course of the disease more closely and adapt the treatment accordingly.

This work was supported by a grant from INSERM (PRC 113025). The manuscript was translated into English by Dr Jessica Blanc.

\section{References}

1 Massion J. Postural changes accompanying voluntary movements. Normal and pathological aspects. Hum Neurobiol 1984;2:261-7

2 Babinski, J. De l'asynergie cérébelleuse. Rev Neurol (Paris) 1899;7:806-16.

3 Martin JP. The Basal Ganglia and Posture. London, Pitman 1967. 152.

4 Gurfinkel VS, Kots JM, Paltsev FI, Feldman AG. In:O Gelfand IM, Gurfinkel VS, Fomin SSV, Tsetlin ML (eds). $\overrightarrow{\mathbb{D}}$ Models of the Structural Functional Organization of Certain Biological Systems. Cambridge, MIT Press, 1971:382-95.

5 Nashner LM, Cordo PJ. Coordination of arm movements and associated postural adjustments in standard subjects. Soc Neurosci Abstr 1980;6:394.

6 Bouisset S, Zattara M. A sequence of postural movements precedes voluntary movements. Neurosci Lett 1981;22:263-70.

7 Cordo PJ, Nashner LM. Properties of postural adjustments associated with rapid arm movements. J Neurophysiol 1982;47:: 287-302.

8 Friedl WG, Hallet M, Simon SR. Postural adjustments associated with rapid voluntary arm movements. 1. Electromyographic data. J Neurol Neurosurg Psychiatry 1984;47:611-22.

9 Marsden CD, Merton PA, Morton HB. Anticipatory postural responses in the human subject. $J$ Physiol (Lond) 1978;275: 47-48P

10 Marsden CD, Merton PA, Morton HB. Human postural responses. Brain 1981;104:513-34.

11 Hugon M, Massion J, Wiesendanger M. Anticipatory postural changes induced by active unloading and comparison with passive unloading in man. Pflügers Arch 1982;393:292-6.

12 Dufosse M, Hugon M, Massion J. Postural forearm changes induced by predictable in time or voluntary triggered unloading in man. Exp Brain Res 1985;60:330-4.

13 Traub MM, Rothwell JC, Marsden CD. Anticipatory postural reflexes in Parkinson's disease and other akinetic-rigid syndromes and in cerebellar ataxia. Brain 1980;103:393-412.

14 Draper IT, Johns RJ. The disordered movement in Parkinsonism and the effect of drug treatment. Bull Johns Hopk Hosp 1964;115:465-80.

15 Evarts EV, Teravainen H, Calne DB. Reaction time in Parkinson's disease. Brain 1981;104:167-86.

16 Yokochi F, Nakamura R, Narabayashi H. Reaction time of patients with Parkinson's disease with reference to asymmetry of neurological signs. J Neurol Neurosurg Psychiatry 1985;48:702-5.

17 Hoehn MM, Yahr MD. Parkinsonism: onset, progression and 
mortality. Neurology 1967:17:427-42.

18 Delong MR. Georgopoulos AP. Motor functions of the basal ganglia. In: Handbook of Physiology. Section 1. The Nervous Sistem. Vol II. Motor Control, Part 2. Brooks VB (ed). Am J Physiol. Bethesda, Maryland 1981;1017-61.

19 Angel RW, Alston W, Higgins JR. Control of movement in Parkinson`s disease. Brain 1970;93:1-14.

20 Cassell K, Shaw K, Stern G. A computerized tracking technique for the assessment of Parkinsonian motor disability. Brain 1973:96:815-26.

21 Flowers KK. Ballistic and corrective movements on an aiming task: intention tremor and Parkinsonian movement disorders compared. Neurology 1975;25:413-21.

22 Bloxham CA, Mindel TA, Frith CD. Initiation and execution of predictable and unpredictable movements in Parkinson's disease. Brain 1984;107:371-84.

23 Hallett M. Khoshbin S. A physiological mechanism of bradykinesia. Brain 1980;103:301-14.
24 Marsden CD. The mysterious motor junction of the basal ganglia: the Robert Wartenberg Lecture. Neurology 1982;32: 514-39.

25 Paulignan Y, Dufosse M, Hugon M, Massion J. Parameters of voluntary movement responsible for the anticipatory adjustment of posture. Neurosci Lett 1986;Suppl.26:5274.

26 Angel RW, Alston W, Garland H. L-Dopa and error correction time in Parkinson's disease. Neurology 1971;21:1255-60.

27 Velasco R, Velasco M. A quantitative evaluation of the effects of L-Dopa on Parkinson's disease. Neuropharmacology 1973;12:89-99.

28 Teravainen H, Calne D. Quantitative assessment of Parkinsonian deficits. In: Parkinson's disease. Current progress, problems and management. Rinne UK, Klinger M, Stamm G (Eds). Elsevier/North-Holland. Amsterdam 1980;145-64.

29 Goldberg G. Supplementary motor area structure and function: review and hypotheses. Behav Brain Sci 1985;8:567-616. 